\title{
Le point sur l'actualité des plates-formes offshore en béton précontraint
}

\author{
Dominique Michel \\ (DORIS Engineering)
}

\section{INTRODUCTION}

Cet article fait le point sur l'état de l'art actuel pour les plates-formes en béton armé et précontraint destinées à l'offshore pétrolier. Il y a actuellement en cours de construction dans le monde 8 plates-formes, et DORIS est impliqué dans 5 plates-formes, une au Canada, deux en Australie, une en Norvège et une en France.

Il ne sera fait état que de 6 de ces 8 plates-formes puisque la barge N'Kossa construite en France fera l'objet d'un exposé au cours de la présente session. Des sept restantes, la plate-forme fixe de Troll, destinée à fournir en gaz l'Europe, est installée sur son site final.

Parmi les plates-formes restantes on décrira :

- Hibernia, plate-forme devant être installée sur les grands bancs de Terre-Neuve et destinée à résister à des icebergs géants.

- Troll Huile, première plate-forme semi-submersible en béton installée en Mer du Nord sur le champ pétrolier de Troll par 325 mètres de fond.
- Heidrun TLP, plate-forme à lignes tendues en béton installée également en Mer du Nord côté norvégien dans 345 mètres d'eau.

- Bream B. et Tuna Ouest, plates-formes fixes destinées à être installées dans 60 mètres d'eau dans le détroit de Bass entre l'Australie et la Tasmanie.

Chacune de ces plates-formes a des caractéristiques bien précises qui seront développées dans les paragraphes suivants.

\section{II $\square$ HIBERNIA}

Cette plate-forme est destinée à développer le champ de pétrole d'Hibernia situé sur les grands bancs de TerreNeuve à $315 \mathrm{~km}$ à l'est de Saint-Jean de Terre-Neuve. Sa grande particularité est de devoir résister à des icebergs géants et à des houles encore plus importantes qu'en Mer du Nord (de l'ordre de 30 mètres de creux). Ces deux particularités en font un ouvrage unique pour lequel seul Doris a trouvé la réponse technique. Ainsi, à titre d'exemple, la pression exercée par la glace et de $600 \mathrm{t} / \mathrm{m}^{2}$.

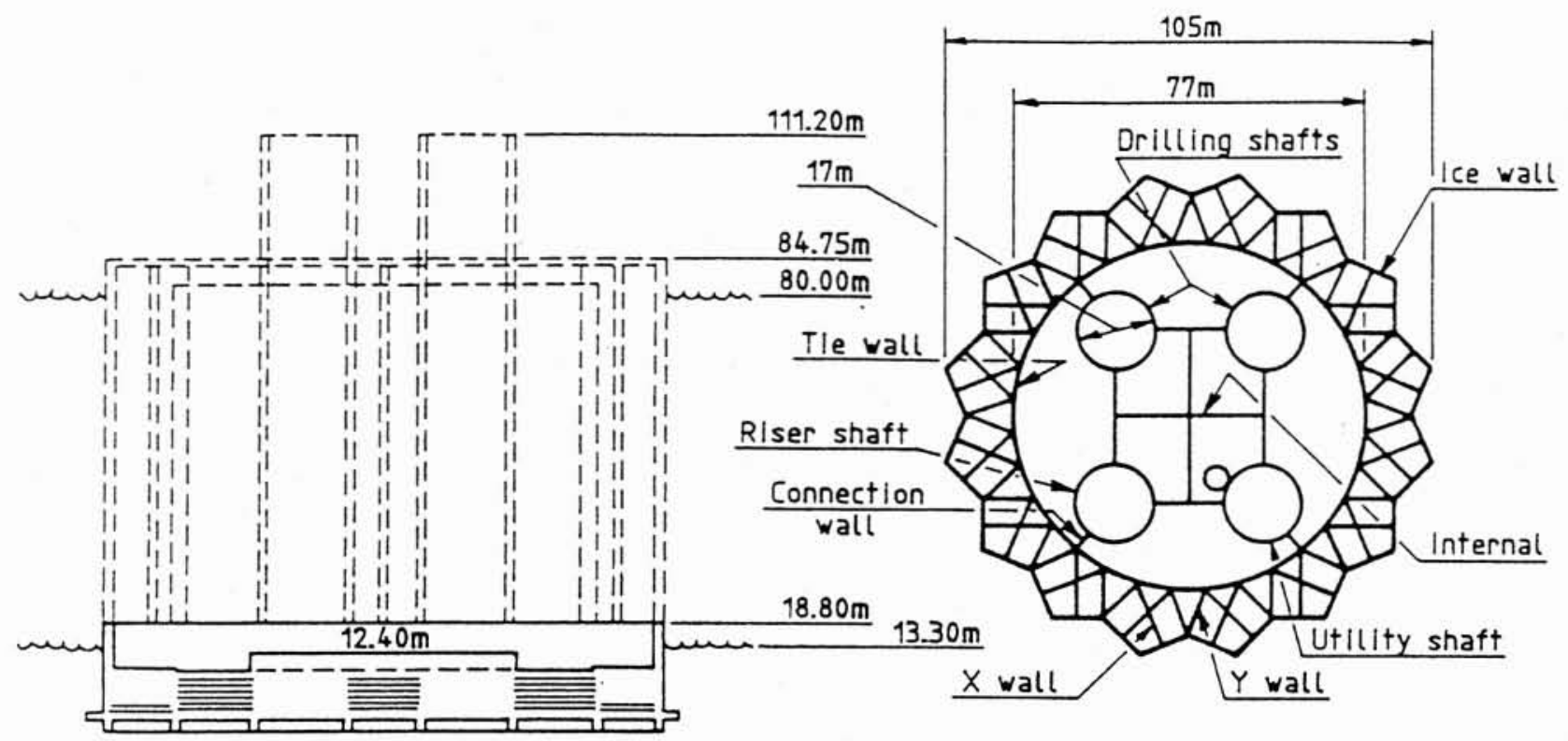

1. Schémas en coupe et élévation de la plate-forme Hibernia. 
Le procédé consiste à protéger la partie intérieure qui stocke le pétrole brut par l'intermédiaire d'un mur antiglace dentelé qui absorbe le choc des icebergs et qui, grâce à des raidisseurs astucieusement disposés, répartit les efforts engendrés sur le cylindre interne.

On retrouve ainsi l'effet voûte connu de nos bâtisseurs de cathédrales dans des temps très anciens.

Pour résumer, ce dispositif, et les dimensions générales de la plate-forme, les schémas en coupe et en élévation donnent des indications utiles.

Les croquis et photos présentés donnent une idée d'ensemble de cet ouvrage gigantesque qui comportera $165000 \mathrm{~m}^{3}$ de béton à haute résistante, développant des résistances de $70 \mathrm{MP}$ sur cylindre à 90 jours. Les additifs et la fumée de silice en font un béton très résistant et d'une bonne ouvrabilité.

Cet ouvrage est construit en trois phases :

- la première dans une cale sèche,

- la deuxième en flottaison,

- la troisième en mer, comprenant le remorquage et l'installation sur le site final par ballastage en eau et ensuite en agrégats lourds.

La première phase est terminée et la plate-forme est en construction dans son site en eau profonde. Elle sera remorquée et installée au printemps 1997 sur le site final, pour une production de pétrole d'environ 125000 barils par jour.

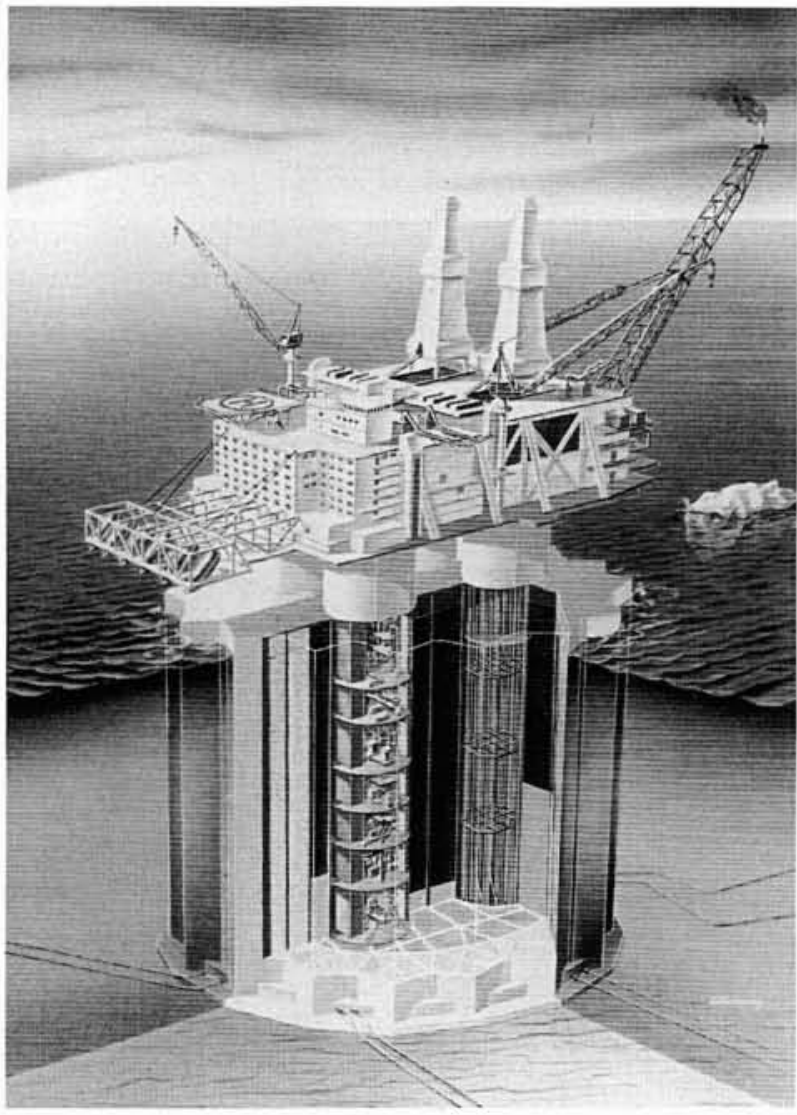

Photo 1. Maquette de la plate-forme Hibernia.

\section{TROLL HUILE}

Dans le monde pétrolier, le pétrole et le gaz sont trouvés de plus en plus souvent dans des profondeurs importantes. Au-delà de 300 mètres d'eau il commence à être intéressant pour cette industrie d'utiliser des supports flottants, qui permettent de s'affranchir des vicissitudes du fond marin. La plate-forme semi-submersible Troll en est un exemple parfait, et est de plus la première plate-forme en béton flottante installée pour produire des hydrocarbures en eau profonde.

Le but de l'ensemble de ce projet est de produire une couche de pétrole de 25 mètres d'épaisseur à l'ouest du champ de gaz géant de Troll, en utilisant des têtes de puits sous-marines et une semi-submersible en béton.

La plate-forme Troll comprend une sous-structure semisubmersible en béton portant des équipements capables de produire 170000 barils par jour de pétrole. Elle est ancrée par des ancrages caténaires dans 325 mètres d'eau. La production venant des puits sous-marins est collectée sur le pont de la sous-structure semi-submersible par des canalisations flexibles. La production est traitée et réexpédiée à terre par les mêmes moyens et à travers une canalisation sous-marine posée sur le fond.

Les principales caractéristiques du projet sont résumées dans le tableau 1.



Photo 2. Maquette de la plate-forme de Troll-Olje. 
Tableau 1. - Principales caractéristiques de la plate-forme Troll.

\begin{tabular}{|l|l|}
\hline Déplacement en opération de la plate-forme & $193000 \mathrm{t}$ \\
Superstructures : ponts et équipements & $L=110 \mathrm{~m}, \ell=60 \mathrm{~m}, H=40 \mathrm{~m}$ \\
Dimensions : & $32500 \mathrm{t}$ \\
Poids en opération : & \\
& \\
Sous-structure & \\
Type: semi-submersible en béton & \\
quantités : & $45000 \mathrm{~m}^{3}$ \\
Béton (C 75) & $16000 \mathrm{t}$ \\
Ferraillage & $3000 \mathrm{t}$ \\
Précontrainte & $101,50 \mathrm{~m} \times 101,50 \mathrm{~m}$ \\
Dimensions en plan & $28 \mathrm{~m} \times 14,50 \mathrm{~m}$ \\
Coupe en travers des pontons & $29 \mathrm{~m}$ \\
Diamètre extérieur des 4 colonnes & $65 \mathrm{~m}$ \\
Hauteur totale de la sous-structure & $40 \mathrm{~m}$ \\
Tirant d'eau & $25 \mathrm{~m}$ \\
Franc-bord & $29 \mathrm{~m}, 12$ à $18 \mathrm{~s}$ \\
Houle centenaire & \\
\hline
\end{tabular}

Doris a été chargée au sein d'un consortium de réaliser le dessin initial et, après ce travail initial de conception, tous les plans de construction de la sous-structure en béton. Il est remarquable de noter qu'à partir de l'avant-projet de Doris les plans de construction et les quantités n'ont pas varié. Ceci est très important pour un engin flottant ayant une grande charge en tête, de façon à lui conserver ses modules de stabilité statique et dynamique.

L’ensemble du génie civil de la sous-structure a été construit dans une cale sèche à côté de Bergen. Les équipements mécaniques, le pont et les modules de production sont installés lorsque la plate-forme est le long d'un quai.

La construction a été amorcée en juin 1993 ; la sousstructure béton a été installée en août 1995.

Les plans et photos présentés ci-après donnent une idée plus exacte de cet ouvrage exceptionnel.

\section{IV $\square$ PLATE-FORME À LIGNES TENDUES D'HEIDRUN}

Parmi les plates-formes flottantes, l'une d'elles présente des particularités intéressantes, c'est la plate-forme à lignes tendues (PLT en français ou TLP en anglais). Elle a pour caractéristiques d'être maintenue en place par des lignes verticales qui sont tendues par la flottabilité du support de surface. On en trouve pour le moment trois dans le Golfe du Mexique, deux en Mer du Nord et une installée cet été en Mer du Nord. C'est celle-ci qui va vous être présentée.

Les croquis 2 et 3 donnent le principe d'un tel ouvrage. En partant de la surface de la mer on trouve:

- La plate-forme supportant le pont et les installations de forage production d'une forme carrée avec 4 pontons dont
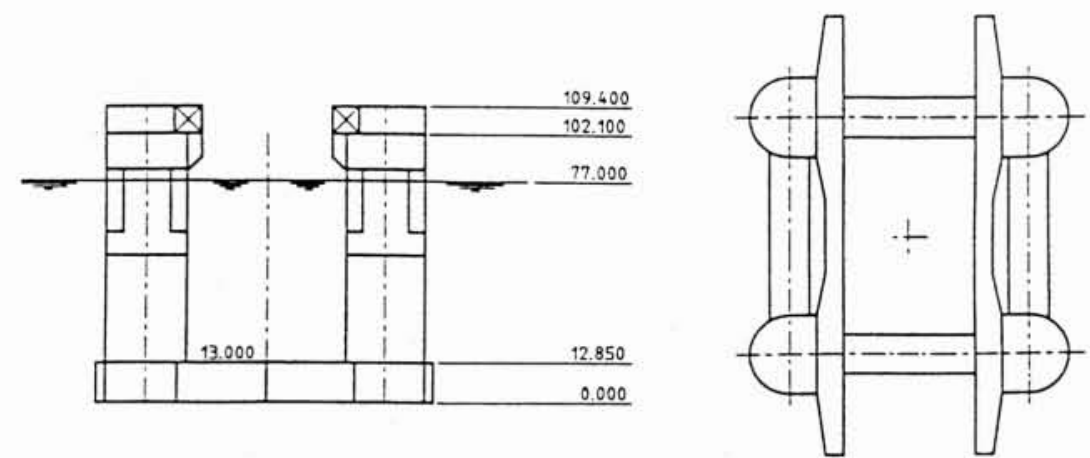

2. Schéma de la plate-forme de Troll-Olje. 
D. MICHEL

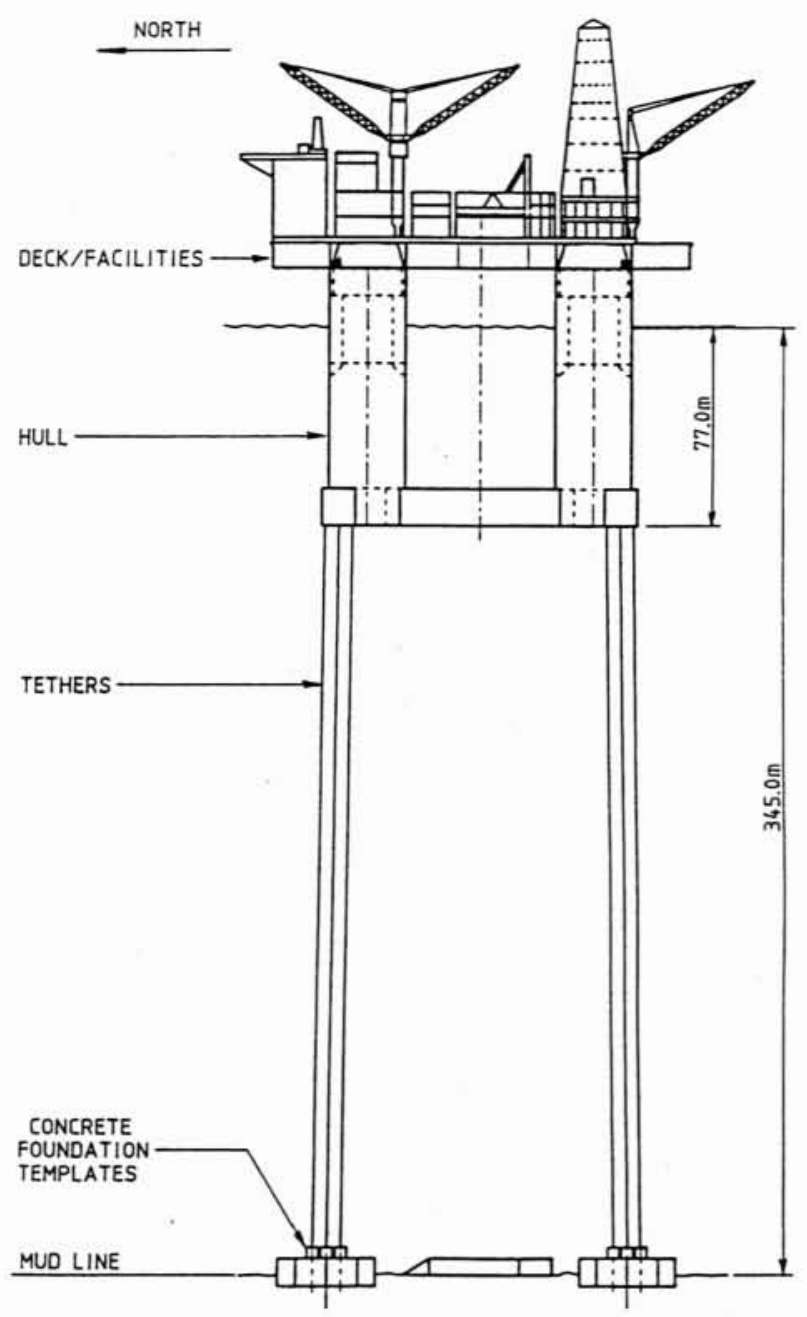

3. Schéma de la plate-forme d'Heidrun.

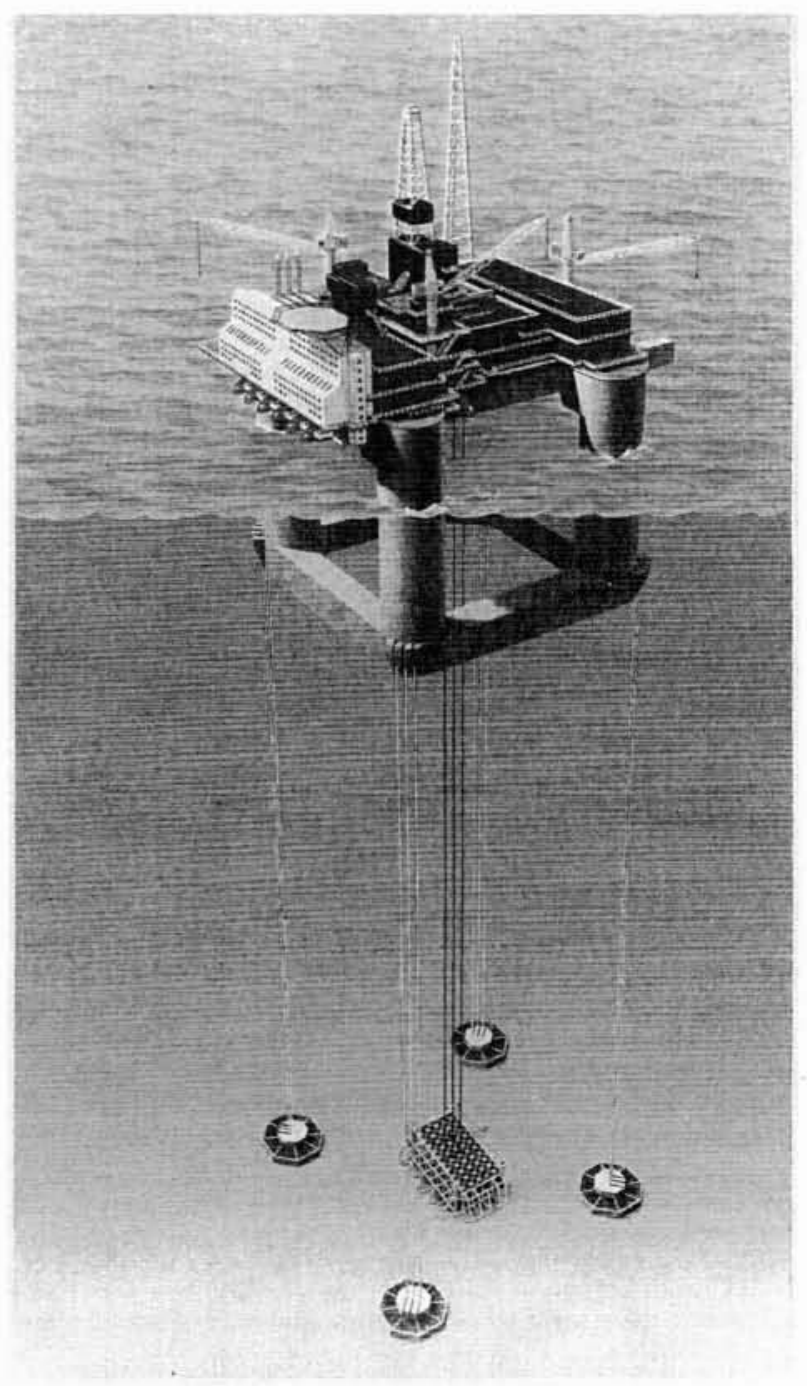

Photo 3. Maquette de la plate-forme Heidrun.

Tableau 2. - Principales caractéristiques de la plate-forme Heidrun.

\begin{tabular}{|l|l}
\hline Poids en tête des équipements et du pont & $59060 \mathrm{t}$ \\
Tension des risers & $6550 \mathrm{t}$ \\
(canalisation qui amène le pétrole sur la plate-forme 56 puits) & \\
Durée de vie de la plate-forme & $50 \mathrm{ans}$ \\
Espacement des colonnes & $80 \mathrm{~m}$ \\
Diamètre des colonnes & $31 \mathrm{~m}$ \\
Dimensions des poutres & $15,95 \mathrm{~m} \times 13 \mathrm{~m}$ \\
Déplacement & $283000 \mathrm{t}$ \\
Tirant d'eau & $77 \mathrm{~m}$ \\
Volume de béton de la coque & $60000 \mathrm{~m}^{3}$ \\
Volume de béton des pontons supportant les modules & $5800 \mathrm{~m}^{3}$ \\
Quantité de ferraillage & $23500 \mathrm{t}$ \\
Quantité de précontrainte & $1700 \mathrm{t}$ \\
Nombre de " tethers" & 16 \\
de diamètre 1,128 m \\
Quantité de béton des fondations & épaisseur: 38 mm \\
& $6760 \mathrm{~m}^{3}$ \\
\hline
\end{tabular}




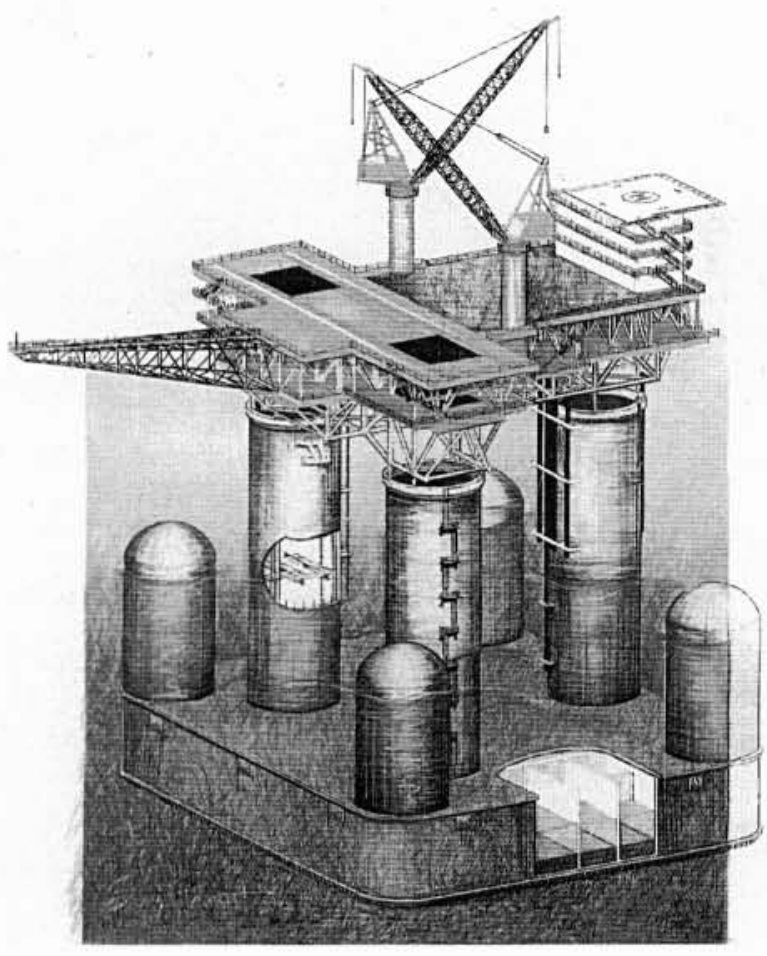

Photo 4. Maquette de la plate-forme Tuna Ouest. la partie inférieure se trouve à $-77 \mathrm{~m}$ et 4 colonnes verticales qui supportent le pont et qui, avec les pontons, donnent la flottabilité pour maintenir en tension les lignes verticales.

- Les lignes verticales appelées « tethers » en anglais qui ont pour but de maintenir la plate-forme en place et de l'empêcher de pilonner.

- Quatre blocs de béton appelés fondations qui sont destinés à reprendre les efforts verticaux statiques et dynamiques du flotteur. Les efforts sont repris par le poids des fondations et par le frottement du sol le long de jupes verticales qui sont enfoncées dans le fond marin par un effet de succion.

La construction de cet ouvrage a commencé début 1993. Le pont et les modules ont été installés en août 1994 et la plate-forme a été posée sur son site final au cours de l'été 1995.

Les caractéristiques principales sont résumées dans le tableau 2.

Le béton pour le flotteur était fabriqué avec des agrégats légers. La densité non ferraillée était de $2 \mathrm{t} / \mathrm{m}^{3}$ et la quantité du C 60.

Pour les fondations, la qualité du béton était du C 75 .

\section{D PLATES-FORMES BREAM B ET TUNA OUEST}

Les deux dernières plates-formes en béton présentées sont remarquables par le fait qu'elles remplacent deux plates-

Tableau 3. - Principales caractéristiques des plates-formes Tuna Ouest et Bream B.

\section{TUNA OUEST}

Dimensions de l'embase

Nombre de jambes

Nombre de flotteurs

Diamètre des jambes et des flotteurs

Poids du pont et des équipements de surface

Volume de béton

Quantités de ferraillage

Quantité de précontrainte

Quantité de ballast solide

Durée de vie de la plate-forme

\section{BREAM B}

Dimensions de l'embase

Nombre de jambes

Nombre de flotteurs

Damètre des jambes et des flotteurs

Poids du pont et des équipements

Volume de béton

Quantités de ferraillage

Quantité de précontrainte

Quantité de ballast solide

Qualité du béton pour ces deux constructions

$92 \mathrm{~m} \times 76 \mathrm{~m} \times 15 \mathrm{~m}$
3
4
$16 \mathrm{~m}$
$7000 \mathrm{t}$
$26000 \mathrm{~m}^{3}$
$9000 \mathrm{t}$
$700 \mathrm{t}$
$17000 \mathrm{t}$
25 ans

$55,5 \mathrm{~m} \times 55,5 \mathrm{~m} \times 15 \mathrm{~m}$
1
3
$12 \mathrm{~m}$
$2000 \mathrm{t}$
$11900 \mathrm{~m}^{3}$
$3700 \mathrm{t}$
$250 \mathrm{t}$
$2500 \mathrm{t}$
C 60


formes métalliques pour le développement d'un champ d'hydrocarbures en Australie.

Ce seront les deux premiers ouvrages construits en béton armé et précontraint sur ce continent.

Elles seront installées dans le détroit de Bass entre l'Australie et la Tasmanie, à $56 \mathrm{~km}$ des côtes et dans 61 mètres de profondeur d'eau. Elles seront construites à Port Kembla au sud de Sydney dans un port en eau peu profonde et leur installation nécessitera un remorquage de 600 kilomètres.

La nouveauté par rapport aux plates-formes classiques en béton tient à ce qu'elles seront construites en eau peu profonde et seront remorquées sur le site final avec tous leurs équipements de pont installés.

Le sol est également une difficulté puisqu'il s'agit de sable carbonaté. Les deux plates-formes seront construites côte à côte d'abord dans une cale sèche et seront terminées en flottaison à quai à l'intérieur du port. Tuna Ouest est une unité de forage-production incluant des quartiers d'habitation, l'ensemble reposant sur trois colonnes tandis que Bream B est une plate-forme puits de forage ayant une seule colonne. Des réservoirs de flottabilité permanents sont disposés aux coins d'une section rectangulaire reposant sur le sol pour permettre une bonne stabilité durant l'immersion sur le site final.
La vue dans l'espace donne une bonne indication de l'architecture de Tuna Ouest.

Les caractéristiques principales de ces plates-formes sont résumées dans le tableau 3.

Le tirant d'eau de ces deux plates-formes pendant le remorquage sur le site final est de $30 \mathrm{~m}$ et de $14,50 \mathrm{~m}$ au maximum dans le port.

Le contrat d'ingénierie de détail de ces deux platesformes a été obtenu par Doris en association avec son partenaire local Kinhill. L'installation de ces deux unités est prévue pour l'année 1996.

\section{VI $\square$ CONCLUSIONS}

On peut voir par les exemples ci-dessus que les platesformes béton, qui sur le plan technique étaient utilisées à poste fixe, se sont étendues pour le flottant.

Par ailleurs, du point de vue géographique, en Mer du Nord s'étaient concentrés la plupart des ouvrages béton et on voit maịntenant que d'autres pays ont adopté ce concept.

Il faut également noter que ce type d'ouvrage présente la meilleure solution dans des environnements de type « arctique». 